Countries with higher incidence were correlated with higher HDI $(r=0.31, p<0.001)$ and a higher prevalence of smoking $(0.26,0.005)$ and overweight $(0.20,0.011$, figure 1$)$. The global ASR of mortality was 1.7. The highest rates were reported in Eastern Asia (ASR=2.4), whilst the lowest rates were found in Middle Africa (0.29). The mortality was the highest in countries with high HDI (1.9) as compared to those with very high (1.5), medium (1.5), and low HDI (0.45). Countries with higher mortality were correlated with higher HDI $(r=0.22, p=0.005)$ and a higher prevalence of smoking $(0.27,0.003)$. No correlations with GDP or alcohol drinking were found $(p>0.05)$.

Conclusions Higher incidence and mortality of gallbladder cancer were found in regions with higher HDI, higher prevalence of smoking and overweight. With population aging and growth, we might expect a further substantial increase in its disease burden, especially for countries with high socioeconomic development. Preventive interventions on reducing the prevalence of risk factors for gallbladder cancer are warranted.

\section{IDDF2020-ABS-0156 ASSOCIATION BETWEEN INCIDENCE AND RISK FACTORS OF LIVER CANCER: A GLOBAL COUNTRY-LEVEL ANALYSIS}

\begin{abstract}
${ }^{1}$ Junjie Huang*, ${ }^{2}$ Harsh K Patel, ${ }^{1}$ Veeleah Lok, ${ }^{1}$ Chun Ho Ngai, ${ }^{1}$ Cedric Chu, ${ }^{3}$ Ping Chen, ${ }^{4}$ Shanjuan Wang, ${ }^{5}$ Lin Zhang, ${ }^{1}$ Xiang-Qian Lao, ${ }^{1}$ Shelly LA Tse, ${ }^{6}$ Wanghong Xu, ${ }^{7}$ ZhiJie Zheng, ${ }^{1}$ Martin Wong. ${ }^{1} J$ ockey Club School of Public Health and Primary Care, Faculty of Medicine, Chinese University of Hong Kong, Hong Kong; ' ${ }^{2}$ Department of Internal Medicine, Ochsner Clinic Foundation, USA; ${ }^{3}$ Department of Gastroenterology, Ruijing Hospital North, School of Medicine, Shanghai Jiaotong University, China; ${ }^{4}$ Department of Gastroenterology, Jiading District Hospital, China; ${ }^{5}$ Melbourne School of Population and Global Health, The University of Melbourne, Australia; ${ }^{6}$ School of Public Health, Fudan University, China; ${ }^{7}$ Department of Global Health, School of Public Health, Peking University, China
\end{abstract}

\subsection{6/gutjnl-2020-IDDF.156}

Background Liver cancer is the sixth most common cancer and the fourth leading cause of cancer mortality globally. The global ASR of incidence of liver cancer was 9.3 per 100,000 persons in 2018. There was an estimated total of 471,000 and 168,200 new cases of liver cancer attributable to HBV and HCV in 2018, respectively. This study aimed to evaluate the association between incidence of liver cancer and its risk factors among 185 countries.

Methods The Age-standardised rates (ASR) for incidence of liver cancer in 2018 were extracted from Global Cancer Observatory (GLOBOCAN). The prevalence of smoking, alcohol consumption, obesity, and diabetes in 2010 for each country were retrieved from the Global Health Observatory (GHO). Primary Outcome: To determine the association between incidence (ASR) and prevalence of risk factors by using multivariable linear regression adjusting for human development index (HDI) and gross domestic product (GDP) per capita.

Results The country with higher incidence was associated with a higher prevalence of smoking (males: $\beta=0.25, p=0.028$ ) and alcohol consumption (females: $\beta=0.94, p=0.042$ ) (table 1: $\beta$, beta coefficient refers to how much does the incidence (ASR) change per unit increase in risk factor). No association between the incidence and body mass index (BMI) or diabetes were found in the current analysis $(p>0.05)$.

Conclusions Smoking and alcohol consumption remain as important risk factors for liver cancer at a country level. Smoking was associated with an increased risk of Country-specific preventive strategies in the reduction of liver cancer burden includes promoting smoking cessation and alcohol control for high-risk populations.

\section{IDDF2020-ABS-0161 MIXED TYPE I AND II GALLBLADDER PERFORATION IN AN ASYMPTOMATIC ELDERLY PATIENT}

${ }^{1}$ Nata Pratama Hardjo Lugito*, ${ }^{1}$ Andree Kurniawan, ${ }^{1}$ Ignatius Bima Prasetya, ${ }^{1}$ Jeremia Immanuel Siregar, ${ }^{2}$ Dewi Purnamasari. ${ }^{1}$ Department of Internal Medicine, Faculty of Medicine, Pelita Harapan University, Indonesia; ${ }^{2}$ Siloam Hospital Lippo Village, Indonesia

\subsection{6/gutjnl-2020-IDDF.157}

Background Gallbladder perforation is a life-threatening event, a severe complication of acute cholecystitis. It is difficult to differentiate to uncomplicated acute cholecystitis. There are three types of gallbladder perforation; (I) acute free perforation into the peritoneal cavity; (II) subacute perforation with pericholecystic abscess; (III) chronic perforation with cholecystoenteric fistula. Type I and II mostly occurred in patients below 50-year-old, while type III in elderly patients with a long history of the biliary stone. We present a case of mixed type I and II gallbladder perforation in an asymptomatic patient.

Methods A 63-years old female with no medical comorbidity was admitted due to watery stool, without nausea, vomiting, abdominal pain or fever. She had a 10 -year history of mild epigastric discomfort. She was normotensive, slightly tachycardia and tachypneu with no fever. There was a mass in the

Abstract IDDF2020-ABS-0156 Table 1 The associations between incidence of liver cancer and its risk factors

\begin{tabular}{|c|c|c|c|c|c|c|c|c|c|}
\hline \multirow{3}{*}{$\begin{array}{l}\text { Outcome } \\
\text { (GLOBOCAN) }\end{array}$} & \multirow[t]{3}{*}{ Risk factor (GHO) } & \multicolumn{4}{|l|}{ Male } & \multicolumn{4}{|c|}{ Female } \\
\hline & & \multirow[t]{2}{*}{$\beta$} & \multirow{2}{*}{\multicolumn{2}{|c|}{$\begin{array}{l}95 \% \\
\mathrm{Cl}\end{array}$}} & \multirow[t]{2}{*}{$p$} & \multirow[t]{2}{*}{$\beta$} & \multirow{2}{*}{\multicolumn{2}{|c|}{$\begin{array}{l}95 \% \\
\mathrm{Cl}\end{array}$}} & \multirow[t]{2}{*}{$p$} \\
\hline & & & & & & & & & \\
\hline Incidence & Smoking & 0.25 & 0.03 & 0.48 & 0.028 & -0.13 & -0.29 & 0.04 & 0.123 \\
\hline \multirow[t]{5}{*}{ (ASR) } & Alcohol drinking & 0.09 & -0.40 & 0.58 & 0.712 & 0.94 & 0.03 & 1.85 & 0.042 \\
\hline & BMI & 0.64 & -0.49 & 1.77 & 0.265 & -0.51 & -1.58 & 0.55 & 0.342 \\
\hline & Diabetes & 0.76 & -0.54 & 2.05 & 0.249 & 0.54 & -0.26 & 1.33 & 0.183 \\
\hline & HDI & -22.7 & -51.5 & 6.07 & 0.121 & -3.54 & -19.2 & 12.2 & 0.656 \\
\hline & GDP & 63.7 & -121.3 & 248.8 & 0.496 & -6.76 & -101.9 & 88.4 & 0.888 \\
\hline
\end{tabular}



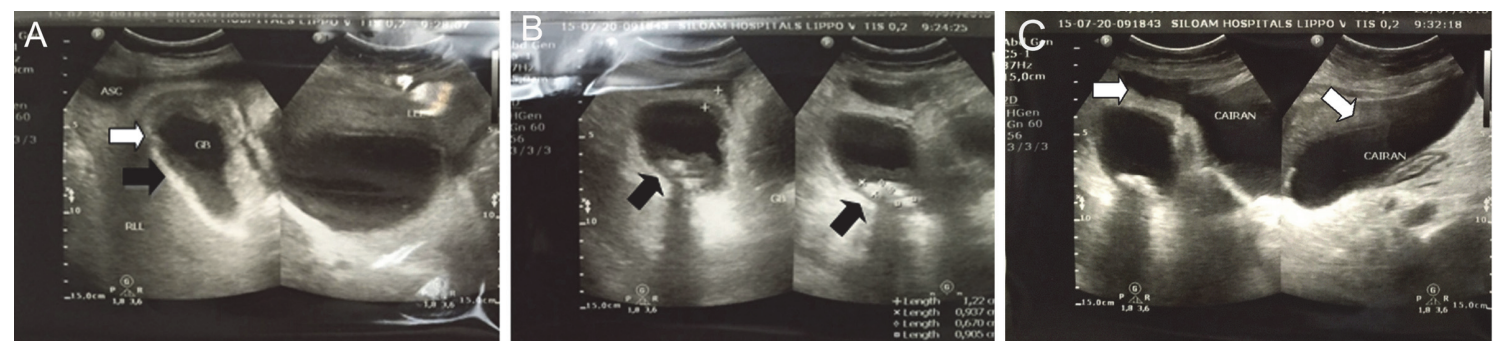

\section{Abstract IDDF2020-ABS-0161 Figure 1}

right hypochondrium diametered 10 centimeters, tender on palpation, but no signs of general peritonitis. Alkaline phosphatase and gamma-glutamyl transferase levels were normal. The ultrasound found distended gallbladder with a thickened wall (figure 1A), gallstones diametered 0.6-1.2 centimeters within sludge (figure 1B) and an anechoic lesion with internal echo outside the liver (figure 1C).

Results On laparotomy, there was dense adhesion between the margin of the right lobe of the liver with anterior peritoneum, and between the omentum with liver, gallbladder and transverse colon, and 500 milliliters of pus within adhesion. The gallbladder was distended with a perforation near fundus, and 8 gallstones were retrieved. Cholecystectomy was then performed. The patient was discharged on the 8th day post-surgery. The patient did not have symptoms and signs suggestive of gallbladder disease or perforation. Location of perforation was at the fundus (type I), but it was sealed by omentum and transverse colon. The sealing contained the bile leak within the extra peritoneal gallbladder fossa thus resulting pericholecystic abscess (type II). Ultrasound and CT scan findings in the setting of gallbladder perforation are pericholecystic fluid collections, gallbladder wall thickening and cholelithiasis.

Conclusions High clinical suspicion of gallbladder perforation based on history, physical examination and ultrasound are sufficient to perform a surgical intervention.

\section{IDDF2020-ABS-0162 TRENDS IN HOSPITALISED DRUG-INDUCED LIVER INJURY FROM 2009 TO 2019 - THE RISE OF NON-PRESCRIPTION MEDICATIONS}

Emily Nash*, Avik Majumdar, Geoffrey McCaughan, Simone Strasser, Ken Liu. Australian National Liver Transplant Unit, Royal Prince Alfred Hospital, Australia

\subsection{6/gutjnl-2020-IDDF.158}

Background Drug-induced liver injury (DILI) is the most common cause of acute liver failure (ALF) in Western countries. We studied the characteristics and outcomes of non-paracetamol DILI.

Methods We retrospectively studied patients admitted to a state-wide quaternary-referral liver transplant (LT) centre for DILI between 2009-2019. Cases were identified by ICD-10 diagnosis code K71. Primary outcome was LT-free survival at 90 days.

Results During the study period, 65 cases of DILI due to non-paracetamol drugs were admitted (57\% female, median age 53). A minority had chronic liver disease (20\%) or psychiatric disease (26\%). The most common implicated drugs are shown in figure 1. Implicated drugs for hospitalised non-paracetamol DILI patients. While overall number of admissions remained stable over time, the proportion due to non-prescription drugs significantly increased $(0 \% \quad 2009-10, \quad 24 \%$ 2011-13, $18 \% \quad 2014-16, \quad 53 \% \quad 2017-19, \quad \mathrm{P}=0.009)$. The

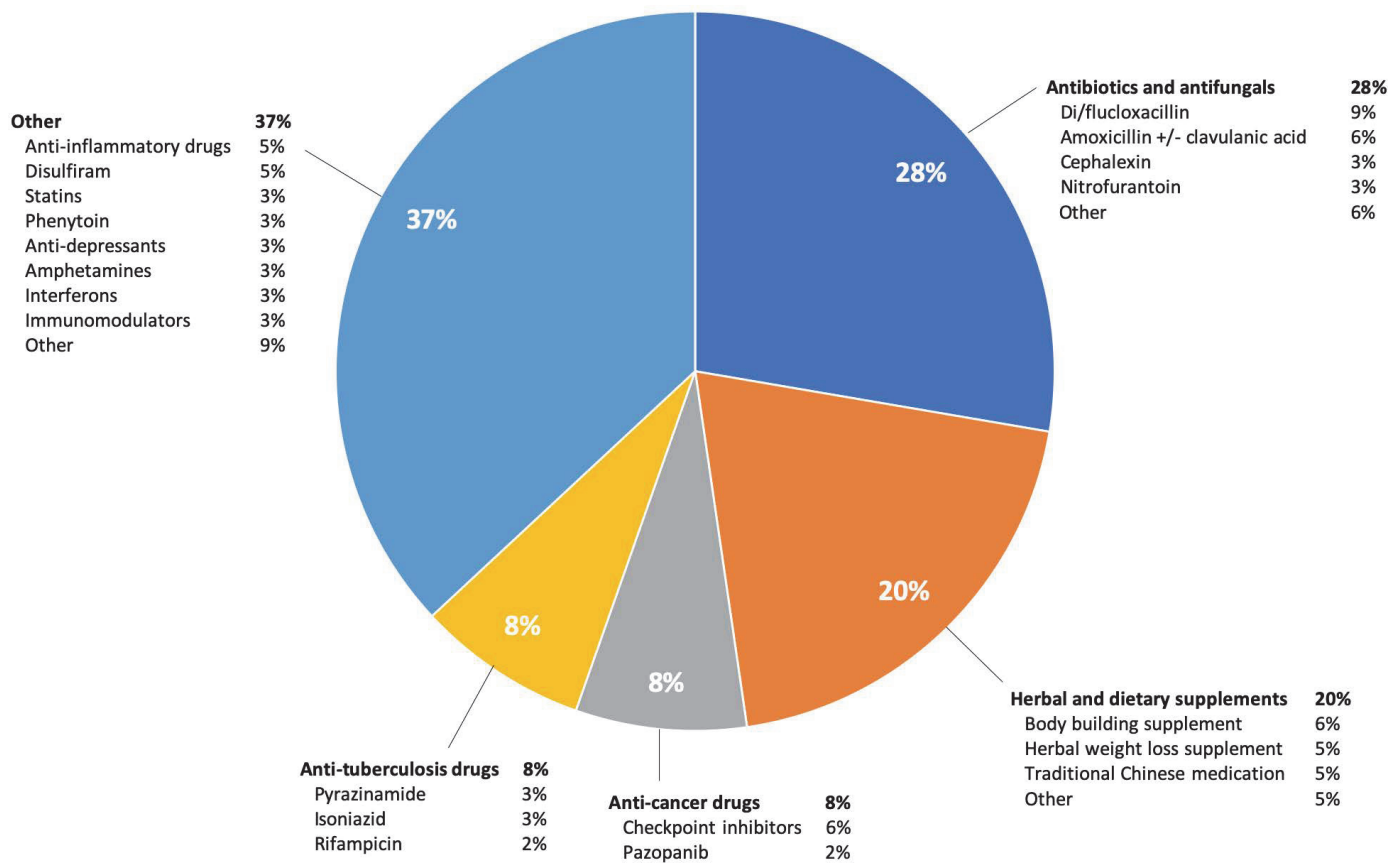

Abstract IDDF2020-ABS-0162 Figure 1 Implicated drugs for hospitalised non-paracetamol DILI patients $(n=65)$ 